\title{
The Case Study of an F/OSS Virtualization Platform Deployment and Quantitative Results
}

\author{
Panagiotis Stathopoulos, Alexandros Soumplis, and Nikos Houssos \\ National Documentation Center/National Hellenic Research Foundation (EKT/NHRF) \\ 48, Vas. Constantinou Av. 11635 Athens, Greece \\ Tel.: +30 2107273996 \\ \{pstath, soumplis, nhoussos\} @ekt.gr
}

In this paper we present practical experiences and results from the deployment of an F/OSS virtualization platform. EKT's (NDC) core IT infrastructure was transformed to a virtualized one, using exclusively F/OSS, while severe budget and timing constraints were in place. This migration was initiated in order to better cope with EKT's services requirements, while accommodating at the same time the need for the in house development of a large scale open access infrastructure. The benefits derived from this migration were not only generic virtualization benefits, such as the quantifiable reduced power consumption and cost reduction through consolidation, but also F/OSS virtualization specific ones.

The creation of the aforementioned open access infrastructure involved extensive software development, employing agile methods. These, were coupled with F/OSS virtualization, in order to meet a number of requirements, which are quite common in a wide area of IT environments and projects. These requirements were: a) the adoption of agile software methodologies, b) the rapidly arising needs for additional specialized small and medium scale applications, c) the in house development of a large number of applications in separated test environments, in parallel with the uninterrupted operation of the production environment, and d) the continuous need for evaluation and experimentation of new platforms and tools, which demanded rapid prototyping in different environments. These requirements demanded the maximum flexibility from the underlying infrastructure, with the proactive, long-term static planning of the required IT infrastructure being especially challenging. Furthermore, due to a combination of budget, timing, cash flow and regulatory constraints the administrative flexibility was severely restricted, with regard to additional equipment and software purchases. When the properties of virtualization were combined with the freedom of F/OSS a significant level of flexibility was achieved, allowing the overall project to be implemented with high quality, while meeting these strict budget, time, and administrative constraints. Similar requirements and conditions exist in a variety of projects, situations and organizations thus making F/OSS a practical candidate for deploying virtualization platforms. Quantitative results, as presented in the poster and a relevant publicly available detailed technical report, indicate that F/OSS virtualization exhibits significant and scalable benefits, in the areas of the total licensing costs, power consumption reduction and overall flexibility achieved. 